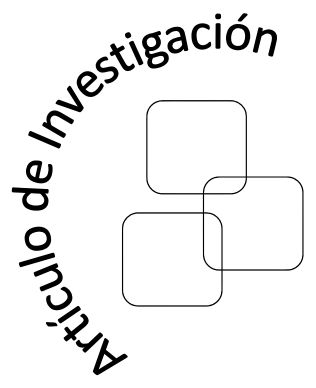

Diego González Herrera Applus-Norcontrol diego.gonzalez.herrera@applus.com

Gustavo Luna Russi Ospina Padilla asociados Ltda. tavorussi@gmail.com

Edwin Rivas Trujillo Universidad Distrital Francisco José de Caldas erivas@udistrital.edu.co
Citación: González D., Luna G. y Rivas, E.(2015). Evaluación del impacto de la generación distribuida mediante índices normalizados con base en la normatividad colombiana y estándares IEEE.

En: Ingeniería, Vol. 20, No. 2, pp. 289-305

(c)Los autores. Titular de los derechos de reproducción: Universidad Distrital Francisco José de Caldas. En línea DOI:

http://dx.doi.org/10.14483/udistrital.jour.reving.2015.2.a08

\section{Evaluación del impacto de la} generación distribuida mediante índices normalizados con base en la normatividad colombiana y estándares IEEE

\author{
Impact assessment of distributed \\ generation using standardized indexes \\ based on colombian regulations and \\ IEEE standards
}

\section{Resumen}

Se presenta una breve revisión de la literatura científica referente a índices normalizados en calidad de potencia y el marco regulatorio colombiano; el trabajo fue motivado por el reto de otros autores de plantear índices para evaluar el impacto de las perturbaciones de calidad de potencia (con enfoque cualitativo y cuantitativo, sin y con generación distribuida). Por ende se presenta una propuesta de índices normalizados (IREG Regulación de tensión, $I S U$ sobretensión y subtensión, $I_{\text {f all a }}$ corriente de falla, $I_{s a g}$ amplitud de hueco de tensión, $I_{T H D V}$ distorsión armónica total en tensión, $I_{T D D}$ distorsión de la demanda total) implementada en un sistema de 34 nodos de IEEE, los cuales son comparados con los propuestos por dichos autores.

Palabras claves: Calidad de potencia, generación distribuida, índices normalizados, regulación colombiana.

\begin{abstract}
This paper considers a short review of the scientific literature concerning standard power quality indexes and the colombian regulatory framework; the work was motivated in other studies proposing indexes to assess the impact of disturbances in power quality (with qualitative and quantitative approaches, with and without distributed generation). Thus, a proposal for standardised indexes is provided (IREG voltage regulation, $I S U$ overvoltage and undervoltage, $I_{\text {f alla }}$ fault current, $I_{s a g}$ amplitude of voltage dip, $I_{T H D V}$ total harmonic voltage distortion, $I_{T D D}$ total demand distortion) implemented within a IEEE 34 nodes system; a comparison with the other proposals is also discussed.
\end{abstract}

Key words: Colombian regulations, distributed generation, indexes, power quality. 


\section{Introducción}

La generación de energía mediante recursos renovables ha llegado a cubrir el $19 \%$ de la demanda energética a nivel mundial para el periodo 2013-2014 [1] [2]. El principal inconveniente de las energías renovables y de los recursos energéticos distribuidos (DER) es que no son competitivos y visibles en el mercado energético [3] [4].

En [5] se define la generación distribuida (GD) como recursos energéticos distribuidos a pequeña escala en redes de baja y media tensión. En la GD pueden surgir problemas técnicos, tales como pérdidas de potencia, regulación de tensión, aumento de niveles de corriente de falla y problemas de calidad como sobretensión, subtensión, armónicos y huecos de tensión entre otros, por lo cual se hacen necesarias herramientas para medir el impacto técnico de la integración de GD.

El Congreso de la República de Colombia expidió en mayo de 2014 la Ley 1715 [6] por la cual regula la integración de las energías renovables no convencionales al sistema energético nacionales, con el fin de reducir el impacto medioambiental (reducción de emisiones de gases de efecto invernadero) y la seguridad del abastecimiento energético. Sin embargo, en la actualidad en materia de regulación no existe un marco regulatorio que contemple la inclusión de la GD en el sistema interconectado nacional.

En este artículo se presenta una breve revisión de la literatura científica referente a índices normalizados en calidad de potencia y el marco regulatorio colombiano, que define la metodología y los límites necesarios para proponer algunos índices que permitan medir el impacto de la generación distribuida en fenómenos como perfil de tensión, sobretensión y subtensión, corrientes de falla, huecos de tensión y armónicos. Finalmente se presenta una comparación entre los índices propuestos y los índices de otros autores, así como los procedimientos utilizados para evaluar el impacto técnico de la GD.

\section{Revisión de índices normalizados y regulación colombiana}

El Congreso de la República de Colombia expidió el 13 de mayo de 2014 la Ley 1715 [6] por la cual regula la integración de las energías renovables no convencionales al sistema energético nacional, con el fin de promover la gestión eficiente de la energía [7], la cual comprende tanto la eficiencia energética como la respuesta de la demanda, a través de instrumentos tributarios, incentivos, mecanismos de cooperación y estímulos para la inversión e investigación para la producción y utilización de fuentes no convencionales de energía.

Los índices normalizados brindan un mecanismo apropiado para establecer rangos y límites que puedan servir como punto de comparación cualitativo y cuantitativo, en cuanto a beneficios y comportamiento de las perturbaciones de calidad de potencia que se pueden presentar con la conexión de generación distribuida. La propuesta de normalización de índices que desarrolla el presente artículo, se basa en una serie de conceptos que plantean diferentes autores en sus propias propuestas de índices de evaluación, que se expondrán en la tabla I. 
Finalmente, con el uso de índices normalizados los autores desarrollan una herramienta útil para analizar el comportamiento de la calidad de potencia de una red de distribución en presencia de generación distribuida.

Tabla I. Trabajos realizados de índices normalizados en calidad de potencia

\begin{tabular}{|c|c|}
\hline Autores y año & Aspectos relevantes \\
\hline $\begin{array}{l}\text { G. Carpinelli P. Ver- } \\
\text { de R. Chiumeo } 2002\end{array}$ & $\begin{array}{l}\text { Se propone un índice de calidad de la energía global (GPQI, } \\
\text { por sus siglas en inglés) para perturbaciones discretas, con ba- } \\
\text { se en indicadores de severidad discretos (DSI, por sus siglas en } \\
\text { inglés). El GPQI se puede clasificar en dos tipologías diferentes. } \\
\text { La primera tipología mide la diferencia entre la forma de onda } \\
\text { ideal de tensión y la forma de onda de tensión real, la segunda } \\
\text { tipología maneja índices tradicionales de calidad de energía para } \\
\text { determinar un valor general para la calidad de tensión [8]. }\end{array}$ \\
\hline V. Gosbell 2002 & $\begin{array}{l}\text { Propone el Índice de Calidad de Energía Unificada (UPQI, por } \\
\text { sus siglas en inglés) donde se tienen en cuenta solo las pertur- } \\
\text { baciones continuas. El UPQI se amplía a las perturbaciones dis- } \\
\text { cretas como caídas de tensión, sobretensiones y transitorios que } \\
\text { parecen ser más críticas para la regulación futura [9]. }\end{array}$ \\
\hline $\begin{array}{l}\text { P. Caramia C. Perna } \\
2006\end{array}$ & $\begin{array}{l}\text { Se plantea el uso de los índices UPQI para evaluar de manera } \\
\text { eficiente la influencia de la generación distribuida sobre la ca- } \\
\text { lidad del suministro, centrándose en armónicos, variaciones de } \\
\text { tensión y huecos [10]. }\end{array}$ \\
\hline $\begin{array}{l}\text { P. Caramia G. Carpi- } \\
\text { nelli A. Russo } 2006\end{array}$ & $\begin{array}{l}\text { Se evalúa el impacto de la instalación de generación distribui- } \\
\text { da introduciendo índices de beneficio, lo que significa, la cuan- } \\
\text { tificación de la influencia de la GD, utilizando el concepto de } \\
\text { porcentaje que es cercano a valores por unidad [11]. }\end{array}$ \\
\hline $\begin{array}{l}\text { A. Bracale P. Ca- } \\
\text { ramia G. Carpinelli } \\
2011\end{array}$ & $\begin{array}{l}\text { Se utilizan los UPQI aplicando el índice de beneficio, para la } \\
\text { evaluación del impacto de la generación distribuida. En este caso } \\
\text { se propone una tabla de decisión basada solo en los resultados } \\
\text { parciales de los nodos de conexión de GD [12]. }\end{array}$ \\
\hline
\end{tabular}

\subsection{Regulación colombiana (calidad de potencia)}

En Colombia el marco regulatorio lo trabajan distintos entidades (Figura 1); dentro de los más importantes se encuentra ICONTEC, que se encarga de la normalización en el país prestando servicios de educación y metrología, entre otros; y la CREG la cual tiene como objetivo principal garantizar la calidad, la cobertura del suministro y expansión de los servicios de energía eléctrica, gas natural y gas licuado. 


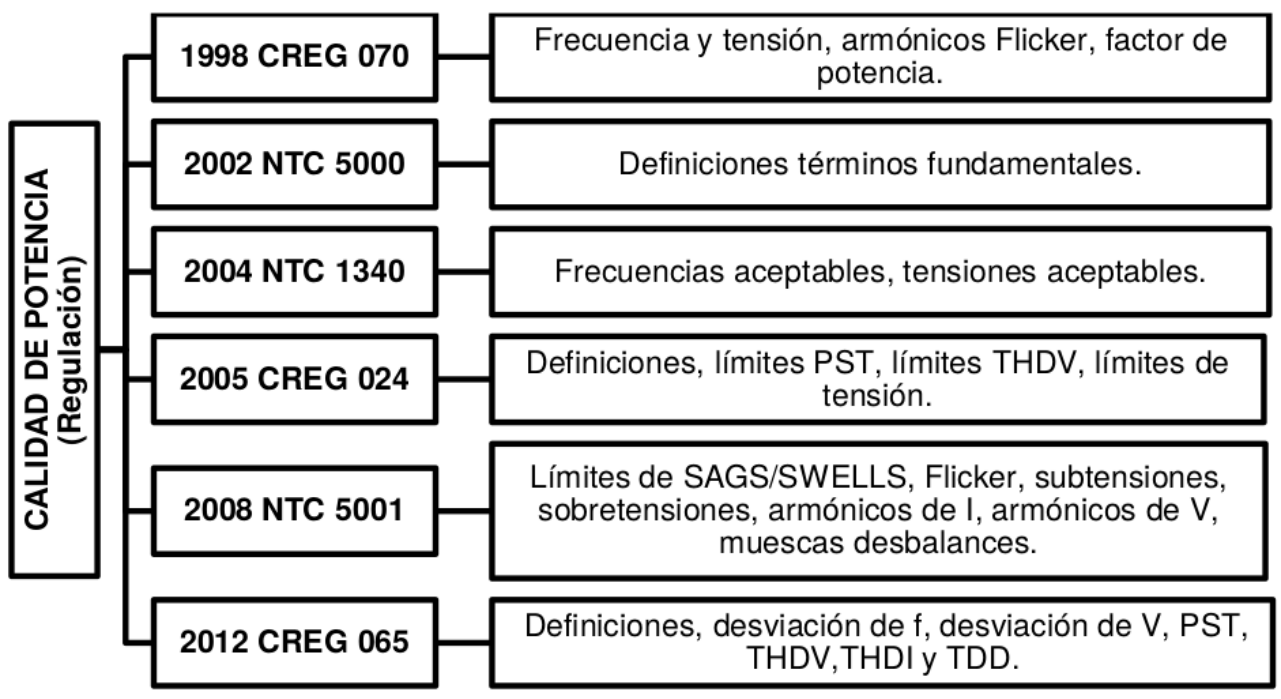

Figura 1. Normatividad relevante colombiana en calidad de potencia.

\section{NTC1340 [13]}

Esta norma (Figura 2) del año 2004 aborda mínimos conceptos de calidad de potencia, sus temáticas centrales son las tensiones y frecuencias nominales en sistema de energía eléctrica en redes de servicio público.

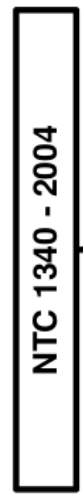

NUMERAL 3 Tabla de Frecuencias aceptables.

Temática: Tensiones y frecuencia nominales en sistemas de energía eléctrica en redes de servicio público. De interés.

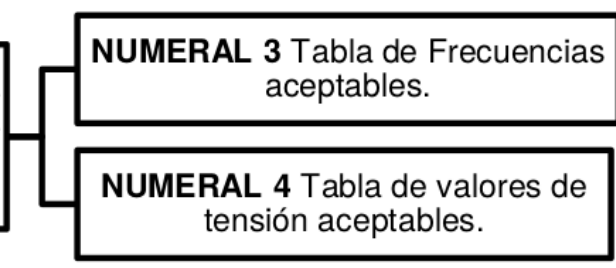

Figura 2. Mapa conceptual NTC 1340-2004.

\section{NTC $5001[14]$}

La NTC 5001 (Figura 3) es la norma técnica colombiana de calidad de potencia eléctrica del año 2008, sus temáticas centrales son establecer los límites y la metodología de evaluación en un punto de conexión común, es una norma que contiene aspectos similares a la norma IEEE 1159. 


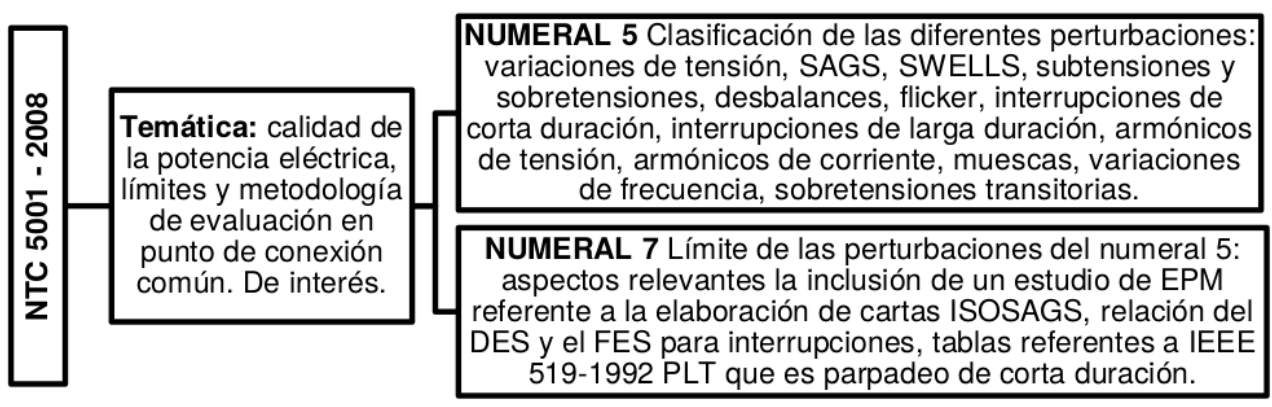

Figura 3. Mapa conceptual de la NTC $5001-2008$.

\section{CREG 070-1998 [15]}

La resolución CREG 070 del año 1998 denominado el reglamento de distribución de energía eléctrica (Figura 4) [15], aborda distintas temáticas como las condiciones de conexión para cargas y generadores, la operación de STR y SDL, equipos de medida, alumbrado público, entre otros. Sin embargo, de gran interés es el Capítulo 6 denominado "Calidad del servicio en los STR y SDL", el cual hace referencia a la calidad de la potencia suministrada, la calidad del servicio prestado, el registro de interrupciones y las políticas para vigilar o supervisar esta calidad del servicio.

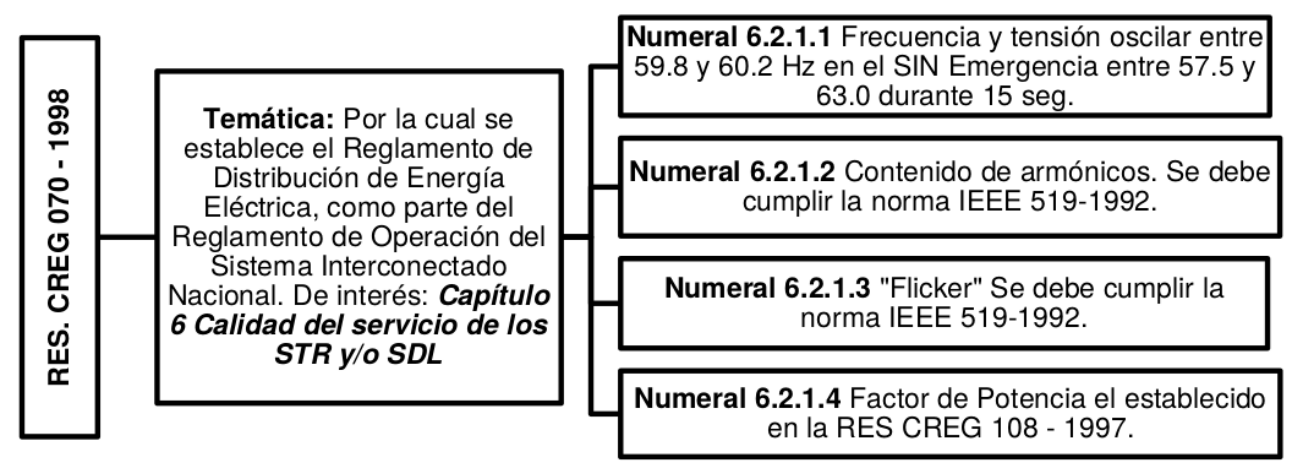

Figura 4. Mapa conceptual CREG 070 - 1998.

\section{CREG 024-2005 [16]}

Esta resolución del año 2005 (Figura 5), hace referencia a las normas aplicables de calidad de potencia eléctrica para los servicios de distribución de energía eléctrica. Su objetivo es darle más importancia a esta temática ya que en la resolución CREG 070 solo es parte de un capítulo de la calidad de potencia eléctrica. Sin embargo, en esta resolución solo se enfoca en algunos fenómenos como lo son el flicker y la distorsión armónica en tensión. 


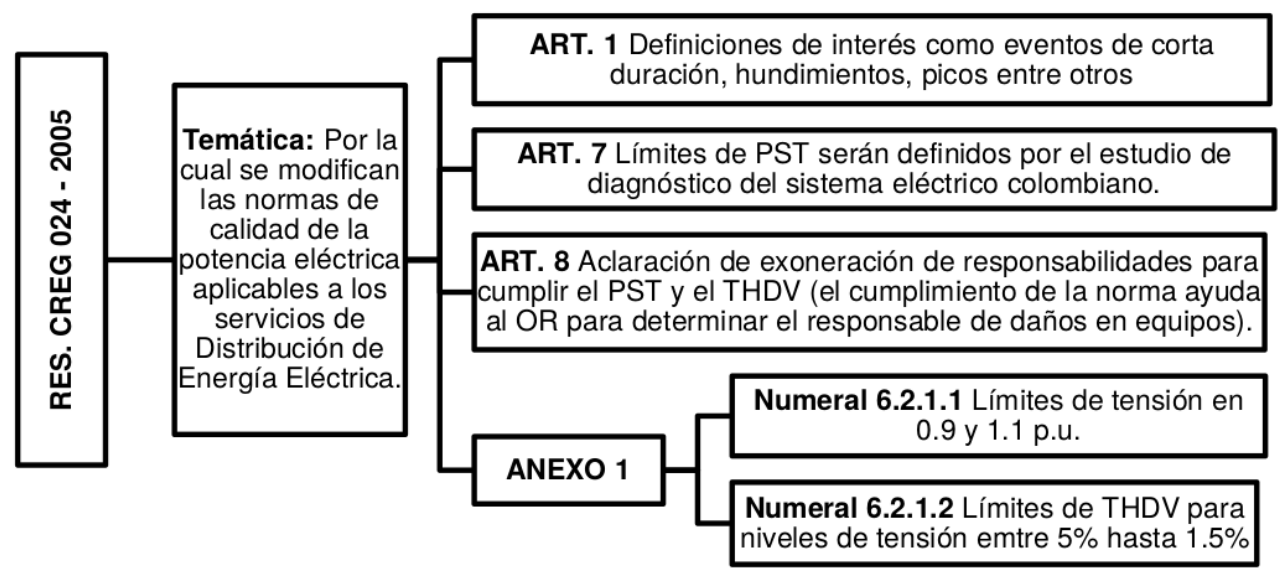

Figura 5. Mapa conceptual CREG 024-2005.

\section{CREG 065-2012 [17]}

La CREG en el año 2012 (Figura 6) lanza este proyecto de resolución el cual trata de complementar las resoluciones anteriores y las compila en una sola, su objetivo es tener de forma clara las políticas de calidad de potencia y ajustarlas para que sean similares a la norma NTC. Esta tiene en cuenta indicadores como desviaciones de frecuencia, desviaciones de tensión, flicker, desbalances y distorsión armónica de tensión y corriente. Además, establece los límites de los indicadores mencionados y la forma en que se deben medir dichos fenómenos.

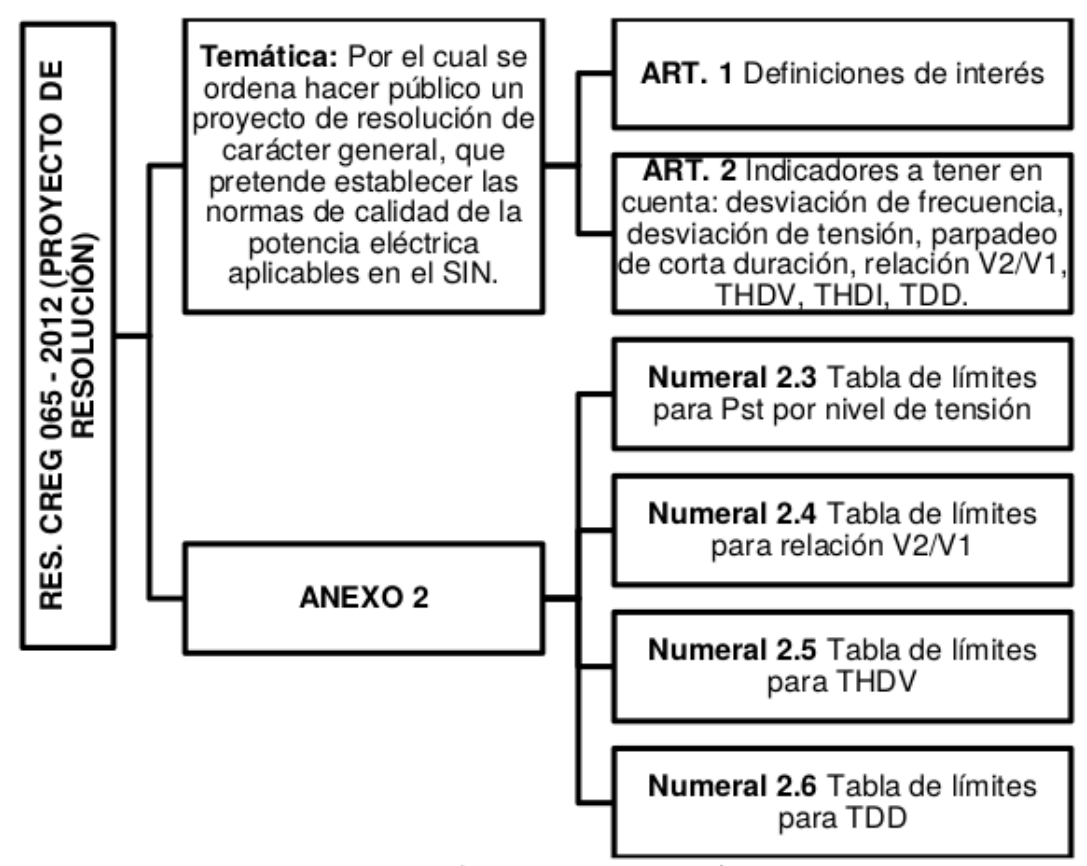

Figura 6. Mapa conceptual CREG $065-2012$. 


\section{3. Índices normalizados propuestos}

Los índices normalizados que se plantean en este artículo surgen de la propuesta de los autores [9] y [11]; dichos índices normalizan cada uno de los disturbios que pueden aparecer en los fenómenos de interés (perfil de tensión, corrientes de falla, huecos de tensión y armónicos), de manera que un índice tiene el valor de 1 cuando se está en el límite de aceptación [9]. Algunos índices cuentan con dos márgenes, superior e inferior, de acuerdo a la normativa relacionada con la perturbación medida y su valor se encontrara entre uno y cero. La ecuación (1) expresa la relación que existe entre la cuantificación del disturbio y su valor límite estandarizado.

$$
I_{n}=\frac{I_{c}-\text { Lim }_{-}}{\operatorname{Lim}_{+}-\text {Lim }_{-}}
$$

Donde $I_{n}$ es el índice normalizado, $I_{c}$ es la cuantificación del evento, $\mathrm{Lim}_{-}$y $\mathrm{Lim}_{+}$son los máximos valores permisibles inferior y superior respectivamente. Cuando se tiene un solo límite $\mathrm{Lim}_{-}$se hace 0 .

El objetivo de los índices normalizados es evaluar el impacto de la presencia de generación distribuida sobre la red eléctrica, en relación con límites establecidos en las normas o por las propias condiciones previas al no uso de la generación distribuida. La evaluación del impacto se hace mediante el índice normalizado de evaluación $I_{E v a l}(2)$, el cual muestra la relación entre los índices normalizados al utilizar $G D$ y los índices normalizados sin utilizar $G D$.

$$
I_{E v a l}=\frac{I_{\operatorname{con} G D}}{I_{\sin G D}}
$$

Donde $I_{\sin G D}$ e $I_{\text {conGD }}$ son la cuantificación normalizada de los eventos sin GD y con GD respectivamente.

\subsection{Perfil de tensión}

El perfil de tensión se evalúa teniendo en cuenta la normativa NTC 1340 [13]/CREG 025 [18]. Define para media tensión, es decir entre $1 k V$ y $62 k V$, una tensión máxima nominal de $+5 \%$ y mínima de $-10 \%$. Además se plantean las siguientes definiciones:

Tensión máxima de un sistema: valor eficaz máximo de tensión que ocurre bajo condiciones de operación normal en cualquier momento y punto del sistema.

Tensión mínima de un sistema: valor eficaz mínimo de tensión que ocurre bajo condiciones de operación normal en cualquier momento y punto del sistema.

De acuerdo a lo anterior se plantean el índice de regulación de tensión (3), el cual considera los dos valores de referencia para la evaluación establecidos por la normativa.

$$
I R E G=\frac{V_{\text {nodo }}-V_{\min }}{V_{\max }-V_{\min }}
$$

Donde, $V_{\text {nodo }}$ es la tensión medida en el punto de conexión común; $V_{\max }$ y $V_{\min }$ son los límites permitidos. 


\subsection{Sobretensión y subtensión}

De acuerdo con NTC5000 [19]/ IEEE 1159 [20] se definen los valores de referencia para el índice de sobretensión y subtensión ISU; además, se muestran las siguientes definiciones:

Sobretensión: variación en estado estable mayor a 1 min, cuyo valor está, por lo menos, $10 \%$ por encima de la tensión nominal del circuito o sistema.

Subtensión: variación en estado estable mayor a 1 min, cuyo valor está, por lo menos, $10 \%$ por debajo de la tensión nominal del circuito o sistema.

En la ecuación (4) se muestra el índice de sobretensión y subtensión $I S U$, que se define como la relación entre la tensión de nodo y la tensión máxima y mínima de referencia para sobretensión y subtensión.

$$
I S U=\frac{V_{\text {nodo }}-V_{\text {lim }_{-}}}{V_{\text {lim }_{+}}-V_{\text {lim }_{-}}}
$$

Donde $V_{\text {lim }}$ es el $10 \%$ de la tensión nominal del nodo por encima para el índice de sobretensión [14]; y $V_{\text {lim }}$ es el $10 \%$ de la tensión nominal del nodo por debajo para el índice de subtensión. Lo anterior con el fin de evaluar la tensión del sistema con respecto a los límites presentados por la norma.

\subsection{Corrientes de falla}

El objetivo de evaluar las corrientes de falla es cuantificar el aumento o disminución de las mismas con la presencia de la generación distribuida. Para esto se utiliza como valor de referencia las corrientes producidas sin GD. En (5) se presenta el índice de corriente de falla $I_{\text {falla }}$

$$
I_{\text {falla }}=\frac{I_{\operatorname{con} G D}}{I_{\sin G D}}
$$

Donde $I_{\sin G D}$ y $I_{c o n G D}$ son las corrientes de falla $\sin G D$ y con $G D$ respectivamente.

\subsection{Huecos de tensión}

De acuerdo a la metodología de evaluación del impacto de la generación distribuida en los huecos de tensión que se presenta en [21], se ha utilizado el índice individual de magnitud.

Los huecos de tensión de acuerdo con NTC 5000 [19] / IEEE 1159 [20] se caracterizan por su amplitud desde 0.1 a 0.9 en p.u. y su duración en instantáneo ( 0.5 a 30 ciclos ) momentáneo (30 ciclos a 3 segundos) y temporal ( 3 segundos a 1 minuto).

Para el índice de amplitud de hueco de tensión $I_{\text {sag }}$ se utiliza la tensión declarada (tensión acordada entre el operador de red y el usuario para ser aplicada en el punto de conexión común. Esta tensión debe estar normalizada NTC 1340 [13]) como referencia fija en el punto de conexión; lo anterior de acuerdo con la NTC 5001 [14] para sistemas de media y baja tensión. 
Índice de amplitud de hueco de tensión $I_{\text {sag }}$, muestra la relación entre la tensión en el nodo del hueco de tensión $V_{\text {nodo }}$ y la tensión de referencia $V_{\text {declarada }}$.

$$
I_{\text {sag }}=\frac{V_{\text {nodo }}}{V_{\text {declarada }}}
$$

La duración del hueco de tensión depende del tiempo de actuación de las protecciones, por lo cual es una característica que ayuda a identificar el evento.

\subsection{Armónicos}

La evaluación de armónicos se realiza mediante la distorsión total armónica, tanto en tensión (THDV) como en corriente (THDI), la cual determina el grado de distorsión de una señal periódica con respecto a la senoidal y se define en términos de la amplitud de los armónicos, como se muestra en las ecuaciones (7) y (8).

$$
\begin{aligned}
& T H D v=\frac{\sqrt{\sum_{h=2}^{50} V_{h}^{2}}}{V_{1}} * 100 \\
& T H D i=\frac{\sqrt{\sum_{h=2}^{50} I_{h}^{2}}}{I_{1}} * 100
\end{aligned}
$$

El índice $I_{T H D V}$ de la ecuación (9) evalúa la THDV, donde THDv $v_{\text {nodo }}$ es la distorsión armónica total en tensión de cada nodo y $T H D v_{\text {limite }}$ es el límite propuesto por la norma NTC 5001 [14] / IEEE519 [20] y que se muestra en la tabla II.

$$
I_{T H D V}=\frac{T H D v_{\text {nodo }}}{T H D v_{\max }}
$$

Tabla II. Valores de referencia de THDv [15].

\begin{tabular}{|c|c|c|}
\hline Rango de tensión & $\begin{array}{l}\text { Distorsión armónica } \\
\text { individual (\%) }\end{array}$ & $\begin{array}{l}\text { Distorsión armónica } \\
\text { total THDv (\%) }\end{array}$ \\
\hline \hline $1 k V<V n<69 k V$ & 3 & 5 \\
\hline $69 k V<V n<161 k V$ & 1,5 & 2,5 \\
\hline$V n>161 k V$ & 1 & 1,5 \\
\hline
\end{tabular}

La evaluación de la distorsión armónica total en corriente (THDI) recurre a otro índice complementario de distorsión total de la demanda TDD ecuación (10); de acuerdo con la norma NTC 5001 [14] / IEEE519 [20], este índice refleja la importancia de las distorsiones armónicas de corriente respecto a la carga máxima del sistema medido, ya que podrían presentarse altas distorsiones de corriente, es decir una medida de THDi elevada, con bajos niveles de carga, que no afectarían en igual proporción al sistema.

$$
T D D=T H D i * \frac{I_{1}}{I L}
$$


Debido a que los límites del TDD, tabla III, están en función de la corriente de corto circuito y la corriente de carga, se presentan diferentes límites en cada uno de los nodos del sistema de pruebas, lo cual dificulta la tarea de normalizarlo. Con base en lo expuesto el propósito del índice de la ecuación (11), es cuantificar el aumento o disminución de la TDD en presencia de generación distribuida, donde $T D D_{n \operatorname{con} G D}$ y $T D D_{n \sin G D}$ son la distorsión total de la demanda de cada nodo con GD y sin GD respectivamente.

$$
I_{T D D}=\frac{T D D_{n \operatorname{con} G D}}{T D D_{n \sin G D}}
$$

Tabla III. Valores límite de distorsión de corriente armónica individual (Di) y de distorsión total de demanda (TDD) en Porcentaje de la corriente de carga (IL)

(Armónicos Impares) [15]

\begin{tabular}{|c|c|c|c|c|c|c|}
\hline \multicolumn{7}{|c|}{ Límites de distorsión en corriente para sistemas de distribución. $120 \mathrm{~V}<\mathrm{Vn} \leq 69 \mathrm{kV}$} \\
\hline Relación Isc/Il & $<11$ & $11 \leq h<17$ & $17 \leq h<23$ & $23 \leq h<35$ & $h \geq 35$ & TDD \\
\hline$<20$ & 4,0 & 2,0 & 1,5 & 0,6 & 0,3 & 5,0 \\
\hline $20<50$ & 7,0 & 3,5 & 2,5 & 1,0 & 0,5 & 8,0 \\
\hline $50<100$ & 10,0 & 4,5 & 4,0 & 1,5 & 0,7 & 12,0 \\
\hline $100<1000$ & 12,0 & 5,5 & 5,0 & 2,0 & 1,0 & 15,0 \\
\hline$>1000$ & 15,0 & 7,0 & 6,0 & 2,5 & 1,4 & 20,0 \\
\hline
\end{tabular}

\subsection{Comparación de índices}

Los índices propuestos para la evaluación de la conexión de la generación distribuida en redes de distribución se ha basado en algunas ventajas que ofrecen los conceptos de los índices UPQI, que han ido evolucionando a lo largo de los últimos años. En la Figura 7 se expone un comparativo entre las características de los índices propuestos y los índices UPQI.

\section{Características de los principales procedimientos de normalización.}

De acuerdo con el artículo Nuevo procedimiento para la normalización de valores numéricos en la toma de decisiones [23] describe las características de los procedimientos utilizados por los índices propuestos y UPQI: 


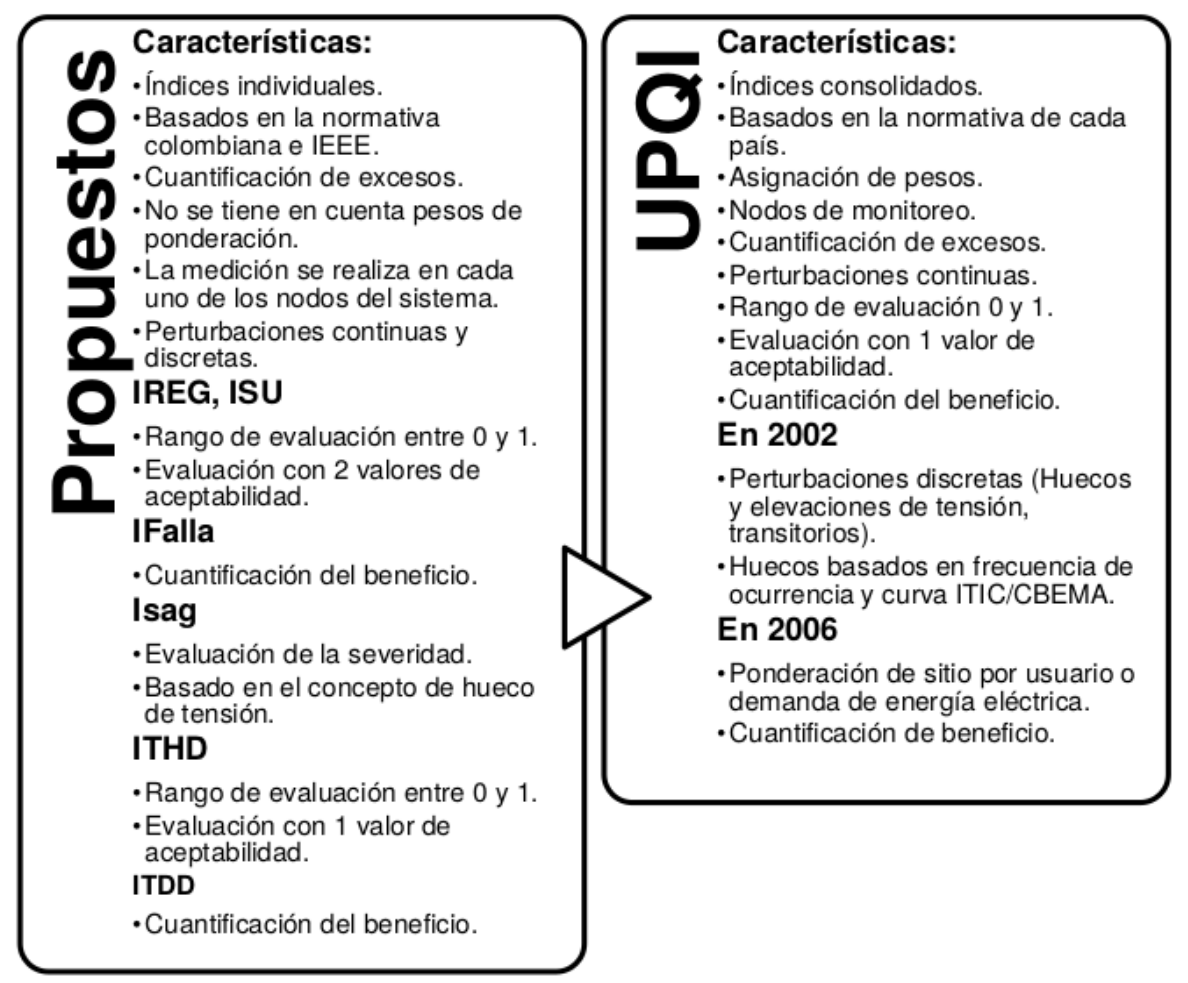

Figura 7. Comparativo de índices Propuestos e UPQI Unifiedpowerqualityindex.

La normalización es la operación mediante la cual un conjunto de valores de una determinada magnitud son transformados en otros, de tal suerte que estos últimos pertenezcan a una escala predeterminada.

La normalización puede realizarse como se ilustra en la (Figura 8):

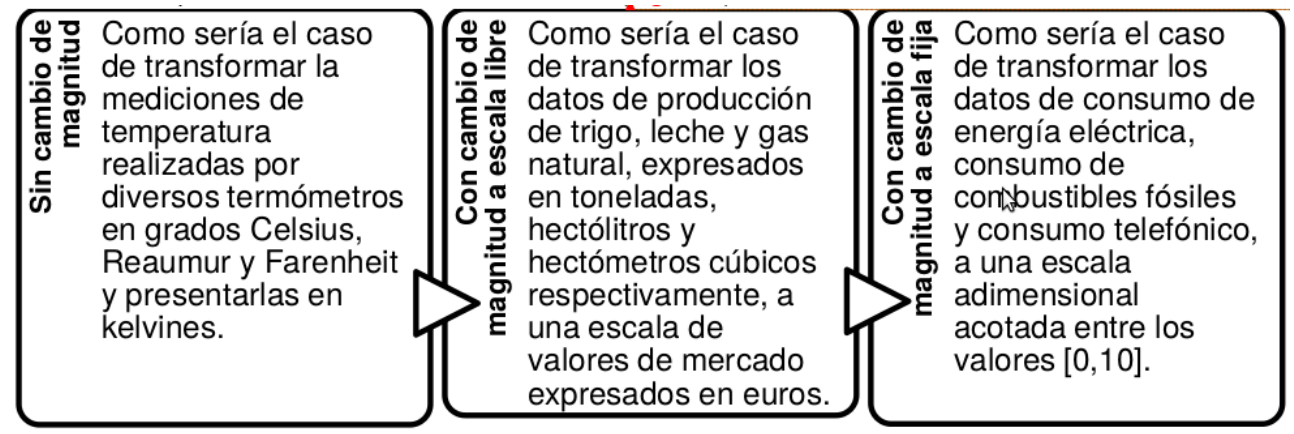

Figura 8. Modos de normalización.

En la Figura 9, se exponen las características de los procedimientos utilizados para la normalización de los índices propuestos y UPQI, con la finalidad de conocer las ventajas y desventajas que matemáticamente podrían presentarse con cada uno de ellos. 


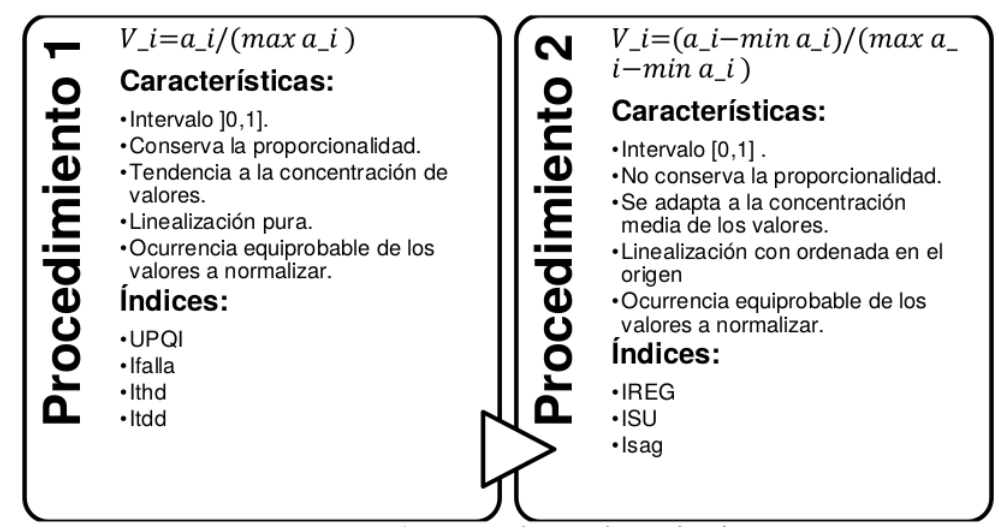

Figura 9. Procedimientos de normalización.

En [23] se aclaran varios aspectos con la concentración de valores: para aquellas ocasiones en las que exista la necesidad de obtener valores normalizados no concentrados, es imprescindible seleccionar un procedimiento de normalización que corrija esta circunstancia. En el caso de la normalización lineal, la condición necesaria para conseguir esta corrección es la proporcionalidad directa entre la concentración de los valores a normalizar y la pendiente de la recta de normalización. De los procedimientos descritos solo el segundo cumple esta condición, ya que la pendiente de la recta de normalización es inversamente proporcional a la amplitud del intervalo de valores y este, a su vez, es inversamente proporcional a la concentración de dichos valores. Quizá por esta característica sea uno de los procedimientos más utilizados en la práctica a pesar de no conservar la proporcionalidad entre los valores originales y los normalizados.

\section{Ejemplo de aplicación de índices normalizados propuestos}

Para evaluar el impacto de la generación distribuida sobre el perfil de tensión, se tomó como caso de estudio el sistema de prueba IEEE 34 nodos (figura 10) el cual es un alimentador real situado en Arizona, con tensión nominal de 24.9 kV [24].

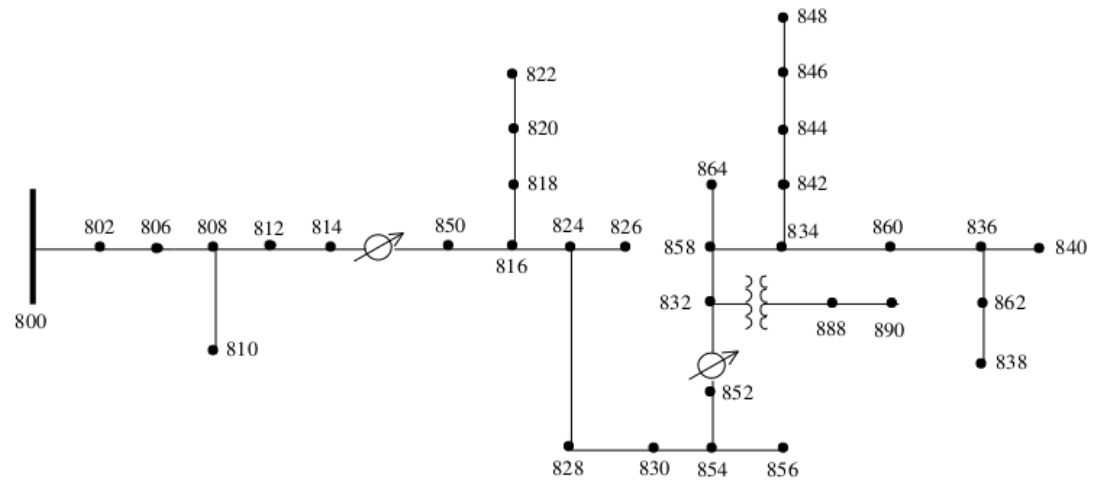

Figura 10. Sistema IEEE de 34 nodos [24]. 
En la tabla IV se presenta el análisis del comportamiento del perfil de tensión con el índice IREG, el cual se realizó a 3312 datos provenientes de 138 nodos durante 24 horas del día.

Tabla IV. Resumen análisis del perfil de tensión índice IREG

\begin{tabular}{|c|c|c|c|c|c|c|c|c|}
\hline GD nodo & Promedio & $\begin{array}{l}\text { Desviación } \\
\text { estándar }\end{array}$ & $\operatorname{Max}(\mathrm{V})$ & Nodo & $\operatorname{Min}(\mathrm{V})$ & Nodo & $\begin{array}{l}\text { Límite máxi- } \\
\text { mo sobrepa- } \\
\text { sado } \\
\%\end{array}$ & $\begin{array}{l}\text { Límite míni- } \\
\text { mo sobrepa- } \\
\text { sado } \\
\%\end{array}$ \\
\hline \multicolumn{9}{|c|}{ Sin GD } \\
\hline & 0,867 & 0,106 & 1,075 & 814.2 & 0,082 & 890.1 & 7,427 & 0 \\
\hline \multicolumn{9}{|c|}{ FP 0} \\
\hline 890 & 0,853 & 0,145 & 1,813 & 890.3 & 0,512 & 852.1 & 14,674 & 0 \\
\hline 832 & 0,871 & 0,103 & 1,139 & 814.3 & 0,067 & 890.1 & 12,983 & 0 \\
\hline 836 & 0,86 & 0,104 & 1,139 & 814.3 & 0,066 & 890.1 & 12,983 & 0 \\
\hline 848 & 0,86 & 0,104 & 1,139 & 814.3 & 0,065 & 890.1 & 12,983 & 0 \\
\hline \multicolumn{9}{|c|}{ FP 1} \\
\hline 890 & 0,881 & 0,086 & 1,334 & 890.1 & 0,598 & $\operatorname{mid} 822.1$ & 12,077 & 0 \\
\hline 832 & 0,872 & 0,102 & 1,088 & 814.3 & 0,066 & 890.1 & 11,715 & 0 \\
\hline 836 & 0,878 & 0,104 & 1,088 & 814.3 & 0,066 & 890.1 & 11,745 & 0 \\
\hline 848 & 0,875 & 0,104 & 1,089 & 814.3 & 0,067 & 890.1 & 11,745 & 0 \\
\hline \multicolumn{9}{|c|}{ FP 0,85 en atraso } \\
\hline 890 & 0,875 & 0,136 & 1,867 & 890.1 & 0,533 & 852.3 & 15,187 & 0 \\
\hline 832 & 0,875 & 0,104 & 1,146 & 814.3 & 0,533 & 890.3 & 13,164 & 0 \\
\hline 836 & 0,875 & 0,106 & 1,146 & 814.3 & 0,533 & 890.3 & 13,225 & 0 \\
\hline 848 & 0,875 & 0,106 & 1,146 & 814.3 & 0,533 & 890.3 & 13,225 & 0 \\
\hline \multicolumn{9}{|c|}{ FP 0,85 en adelanto } \\
\hline 890 & 0,875 & 0,097 & 1,075 & 814.2 & 0,28 & 890.3 & 6,129 & 0 \\
\hline 832 & 0,875 & 0,101 & 1,075 & 814.2 & 0,075 & 890.1 & 7,82 & 0 \\
\hline 836 & 0,875 & 0,103 & 1,075 & 814.2 & 0,076 & 890.1 & 7,85 & 0 \\
\hline 848 & 0,875 & 0,103 & 1,075 & 814.2 & 0,077 & 890.1 & 7,911 & 0 \\
\hline
\end{tabular}

A manera de ejemplo en la ecuación (12) se ilustra el cálculo del índice de regulación en el nodo 824 fase 2 en la hora 2 del perfil de tensión.

- Tensión $\sin \mathrm{GD}=14680,05 \mathrm{~V}$

- Tensión nominal de fase $=14376,02 \mathrm{~V}$

- $V_{\max }(+5 \%$ de la tensión nominal $)=15094,80 \mathrm{~V}$

- $V_{\min }(-10 \%$ de la tensión nominal $)=12938,40 \mathrm{~V}$

- $V_{\text {min }}=14680,05 \mathrm{~V}$

$$
R E G=\frac{14680,05 \mathrm{~V}-12938,40 \mathrm{~V}}{15094,80 \mathrm{~V}-12938,40 \mathrm{~V}}=0,807 \mathrm{pu}
$$

El resultado obtenido de 0,807 en $p . u$. en la ecuación (12), se encuentra en el rango entre 0 y 1 y por lo tanto no alcanza o sobrepasa ninguno de los límites permisibles. 


\section{1. Índice de regulación IREG sin GD}

En la figura 11 se muestra de manera general el perfil en todos los nodos del sistema al aplicar el índice IREG sin GD.

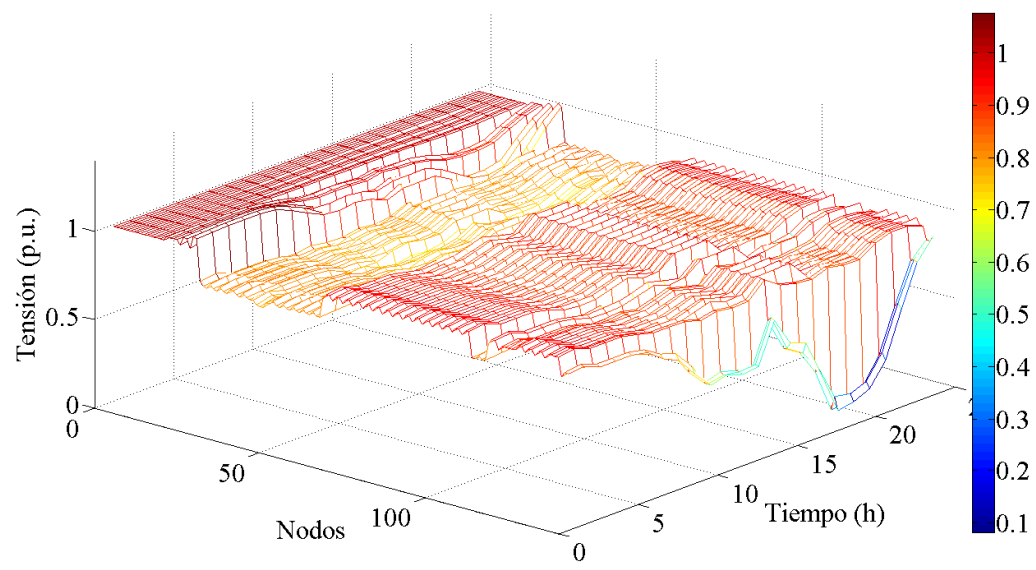

Figura 11. Perfil de tensión sin GD índice IREG.

La Tabla IV se muestra que sin GD solo en el 7,42\% de las veces se sobrepasa el límite máximo aceptable y su valor llega a 1,075. Asimismo, los nodos que han superado la máxima tensión permisible son los cercanos al nodo infinito durante las primeras horas del día, incluso el nodo fuente supera el límite durante el mayor tiempo del día (18 horas). Cabe añadir que los valores más cercanos a 0 se registran en el nodo 890 que corresponde al nivel de 2401, $77 \mathrm{~V}$.

\section{2. Índice de regulación IREG con GD}

En la Tabla IV se muestra que el caso con el mejor promedio 0,853 p.u. en comparación con el escenario sin GD se encuentra conectando GD en el nodo 890 con un factor de potencia 0; sin embargo se supera el límite aceptable 1,81 p.u. y superando el límite máximo aceptable $14,67 \%$ volviéndolo un caso crítico.

El mayor incremento en la tensión (1,86 p.u) ocurre en el nodo 890 fase 1 cuando se conecta el generador en el mismo nodo 890 con factor de potencia 0,85 en atraso; es decir, la tensión de fase alcanza 2834,10 V cuando la tensión nominal es de 2401,77 V. En la Figura 12 se muestra el perfil de tensión en el nodo 890 en la fase 1.

La mejor opción se presenta al conectar GD en el nodo 890 con factor de potencia en adelanto, debido a que se mantiene el sobrepaso del límite superior aceptable del escenario sin GD 1,075 p.u. y su ocurrencia baja del 7,42\% el 6,12\%, además se alejó del límite inferior que paso de 0,08 p.u. a 0,28 p.u. Dicho de otra manera, fue el caso donde menos se afectó el perfil de tensión. 


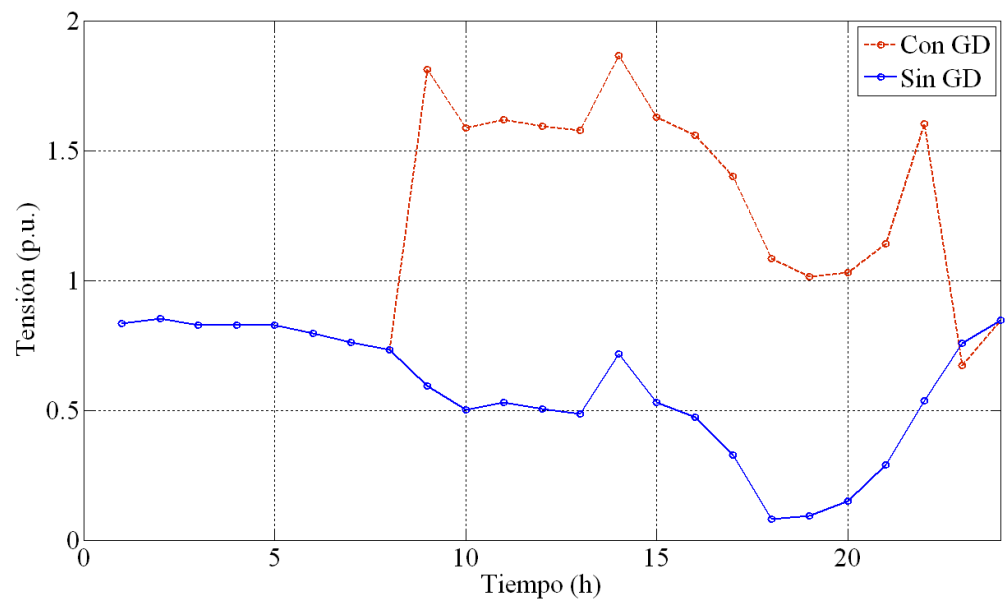

Figura 12. Perfil nodo 890.1 , GD en $890 \mathrm{FP}=0.85$ atraso. Caso crítico.

El caso óptimo se presenta cuando se conecta el generador con factor de potencia 0,85 en adelanto en el nodo 890. Lo ideal, sería un perfil de tensión en donde todos los nodos del sistema tuvieran como tensión real la misma tensión nominal; de acuerdo con el índice IREG la tensión nominal del sistema es 0,666 p.u. con un promedio en los datos de 0,666 p.u. y una desviación estándar cero.

\section{Conclusiones}

La contribución al caso colombiano se hace con la aplicación en un entorno GD por medio del índice de evaluación, el cual sirve como aporte de la Ley 1715 del 2014 [6] de Colombia reflejada en el Artículo 8 sobre la promoción de la autogeneración a pequeña y gran escala y la generación distribuida. En materia de regulación no existe un marco regulatorio que contemple la inclusión de la GD en el sistema interconectado nacional, por lo tanto algunos resultados no pueden ser aclarados completamente, ni se puede evidenciar la utilidad de la inclusión de GD. A parte del marco regulatorio, se requiere más investigación sobre la tecnología de la generación distribuida y el nivel de penetración de la misma.

Para evaluar el impacto (con enfoque cualitativo y cuantitativo, sin y con generación distribuida), basados en la normatividad de las perturbaciones de calidad de potencia (perfil de tensión, sobretensiones, corrientes de falla, huecos de tensión y armónicos), se propusieron los índices normalizados (IREG Regulación de tensión, ISU sobretensión y subtensión, $I_{\text {falla }}$ corriente de falla, $I_{s a g}$ amplitud de hueco de tensión, $I_{T H D V}$ distorsión armónica total en tensión, $I_{T D D}$ distorsión de la demanda total).

Debido a que la normativa colombiana e IEEE no define límites generales y específicos para las corrientes de cortocircuito, la evaluación solo se realiza con base en un índice cualitativo. + Es poco viable generalizar un índice normalizado para distorsión total de la demanda (TDD), ya que en el sistema IEEE 34 nodos cada nodo se cuenta con un límite de distorsión distinto; lo anterior se debe a que el TDD depende de la corriente de corto circuito y la corriente de demanda. 


\section{Referencias}

[1] "Renewable power generation costs in 2014", IRENA. 2014

[2] "Renewables 2014, global status report", REN-21. 2014

[3] H. Saboori, M. Mohammadi and R. Taghe, "Virtual Power Plant (VPP), Definition, Concept, Components and Types", in Power and Energy Engineering Conference (APPEEC), 2011 Asia-Pacific, pp.1-4, 25-28 March 2011.

[4] K. El Bakari and W. L. Kling, "Development and operation of virtual power plant system", in Innovative Smart Grid Technologies (ISGT Europe), 2011 2nd IEEE PES International Conference and Exhibition on, pp.1-5, 5-7 Dec. 2011.

[5] L. I. Dulau, M. Abrudean and D. Bica, Ïmpact of distributed generation upon Reghin 'Lăpuşna Medium Voltage line", in Fundamentals of Electrical Engineering (ISFEE), 2014 International Symposium on , vol., no., pp.1-5, 28-29 Nov. 2014.

[6] Congreso de la República de Colombia, Ley 1715 de 2014, Bogotá D.C., 2014.

[7] J. E. S. Haan, P. H. Nguyen, W. L. Kling and P. F. Ribeiro, "Social interaction interface for performance analysis of smart grids", in Smart Grid Modeling and Simulation (SGMS), 2011 IEEE First International Workshop on, pp.79-83, 17-17 Oct 2011.

[8] G. Carpinelli, P. Caramia, P. Varilone, P Verde, R. Chiumeo, I. Mastrandrea, F. Tarsia and O. Ornago, . ${ }^{\text {A global }}$ index for discrete voltage disturbances", in Electrical Power Quality and Utilisation, 2007. EPQU 2007. 9th International Conference on, pp.1-5, 9-11 Oct. 2007.

[9] V. J. Gosbell, B. S. P. Perera and H. M. S. C. Herath, Ünified power quality index (UPQI) for continuous disturbances", in Harmonics and Quality of Power, 2002. 10th International Conference on, vol.1, pp.316-321, 6-9 Oct. 2002.

[10] P. Caramia, C. D. Perna, P. Verde, F. Buriola, A. Stocco and R. Turri, "Power Quality Indices of Distribution Networks with Embedded Generation", in Universities Power Engineering Conference, 2006. UPEC '06. Proceedings of the 41st International, vol.3, pp.1026-1030, 6-8 Sept. 2006.

[11] P. Caramia, G. Carpinelli, A. Russo and P. Verde, "Power Quality Assessment in Liberalized Market: Probabilistic System Indices for Distribution Networks with Embedded Generation", in Probabilistic Methods Applied to Power Systems, 2006. PMAPS 2006. International Conference on, pp.1-6, 11-15 June 2006.

[12] A. Bracale, P. Caramia, G. Carpinelli, A. Russo and P. Verde,, "Site and System Indices for Power-Quality Characterization of Distribution Networks With Distributed Generation", in Power Delivery, IEEE Transactions on, vol.26, no.3, pp.1304-1316, July 2011.

[13] ICONTEC, Norma Colombiana NTC 1340 Electrotécnia, Tensiones y frecuencia nominales en sistemas de energía eléctrica en redes de servicio público, Colombia, 2004.

[14] ICONTEC, Norma Colombiana NTC 5001 Calidad de la potencia eléctrica. Límites y metodología de evaluación en punto de conexión común, Colombia, 2008.

[15] CREG, Resolución Creg 070 de 1998, Colombia.

[16] CREG, Resolución Creg 024 de 2005, Colombia.

[17] CREG, Regulación Creg 065 de 2012, Colombia.

[18] CREG, Resolución Creg 025 de 1995, Colombia.

[19] ICONTEC, NTC 5000 Calidad de la potencia eléctrica (CPE), Definiciones y términos fundamentales, Colombia, 2002.

[20] IEEE. "Recommended Practice for Monitoring Electric Power Quality", in IEEE Std 1159-2009 (Revision of IEEE Std 1159-1995),pp.c1-81, June 262009.

[21] Li, Zheng, Yao, Zhang and Lin Lingxue, "Studies on Voltage Sag in Distribution Network Containing Distributed Generations", in Power and Energy Engineering Conference (APPEEC), 2012 Asia-Pacific, pp.1-5, 27-29 March 2012.

[22] ÏEEE Recommended Practices and Requirements for Harmonic Control in Electrical Power Systems", in IEEE Std 519-1992, pp.1-112, April 91993

[23] V. Cloquell, M. Santamarina and A. Hospitaler, "Universidad de Zaragoza: Nuevo procedimiento para la normalización de valores numéricos en la toma de decisiones", 2001. [En línea]. Available: http://www.unizar.es/aeipro/finder/ORGANIZACION \%20Y \%20DIRECCION/DD18.htm 
[24] W. H., Kersting, Radial distribution test feeders", in Power Systems, IEEE Transactions on, vol.6, no.3, pp. 975-985, Aug 1991.

\section{Diego González Herrera}

Ingeniero Eléctrico de la Universidad Distrital Francisco José de Caldas, Ha laborado como ingeniero de proyectos y actualmente labora con Applus Norcontrol.

e-mail: diego.gonzalez.herrera@applus.com

\section{Gustavo Luna Russi}

Ingeniero Eléctrico de la Universidad Distrital Francisco José de Caldas, Ha laborado como ingeniero de proyectos y actualmente es ingeniero de proyectos en Ospina Padilla Asociados.

e-mail: tavorussi@gmail.com

\section{Edwin Rivas Trujillo}

Profesor Titular Universidad Distrital Francisco José de Caldas, Ingeniero Electricista, Magister en Sistemas de Generación de Energía Eléctrica, Máster en Ingeniería Eléctrica, Electrónica y Automática y Doctor en Ingeniería Eléctrica, Electrónica y Automática, Director del grupo de Investigación de Compatibilidad e Interferencia Electromagnética GCEM.

e-mail: erivas@udistrital.edu.co 